\title{
Stroke and poor therapeutic adherence in giant cell arteritis: A case report
}

\author{
Jamir Pitton Rissardo, Ana Letícia Fornari Caprara \\ Medicine Department, Federal University of Santa Maria, RS, \\ Brazil Federal University of Santa Maria Health Sciences Center, Brazil
}

\begin{abstract}
Giant cell arteritis (GCA) is an inflammatory disease involving mainly the cranial arteries branches. We present a case of an old male with right hemiparesis that was admitted to our hospital. The subject's daughter stated that the patient had gone to another clinic 3 months ago because he experienced anorexia and temple headache. A temporal artery biopsy proved GCA. Prednisone was started. Within one month, the prednisone-dose was $40 \mathrm{mg} / \mathrm{day}$. The patient was asymptomatic, but the prednisone-dose was maintained because he had elevated inflammatory markers. On admission, the subject had right limbs weakness and global-aphasia with six hours of onset. Twelve hours after admission, the patient developed a seizure, and a CT-scan showed a hypodense area in the territory of the left middle cerebral artery. Upon further questioning, the subject's family admitted that he reduced the dose of prednisone of $40 \mathrm{mg} /$ day to $10-20 \mathrm{mg} /$ day without any medical advice 2 -weeks after the stroke.
\end{abstract}

Keywords: stroke, giant cell arteritis, steroids

\section{INTRODUCTION}

Giant cell arteritis (GCA) is an inflammatory disease involving most commonly the cranial branches of arteries that originate from the aortic branch. About three percent of patients with GCA will have a stroke between the onset of symptoms of the disease and four weeks after the onset of corticosteroid therapy (1). However, to the author's knowledge none case of stroke secondary to corticoid therapy irregular use in GCA has been reported. Herein, we report a case of an older man who was diagnosed with GCA and probably due to poor adherence to medication had a stroke.

\section{CASE REPORT}

A 74-year-old male presenting with right hemiparesis within six hours of onset was admitted to our hospital. The subject's daughter stated that her father had gone to another clinic three months ago because he had experienced anorexia and left tem- ple headache. In the physical examination was observed absent pulse in a tender and enlarged left temporal artery. The temporal artery biopsy showed panarteritis and giant cells without fibrinoid necrosis. Laboratorial tests were within the normal limits, except by elevated erythrocyte sedimentation rate and C-reactive protein (Table 1). A brain MRI was normal. The patient started prednisone $1 \mathrm{mg} /$ $\mathrm{Kg}$. Two weeks after he was asymptomatic and the prednisone was reduced to $50 \mathrm{mg} /$ day, another two weeks and the dose was reduced to $40 \mathrm{mg}$ /day. The patient was asymptomatic, but the dose was maintained because the inflammatory markers still were elevated.

On admission, the subject's daughter reported that her father had right upper and lower limb weakness and aphasia within six hours of onset. He was a retired farmer and his family history was negative for cerebro and cardiovascular disease. The neuropsychological assessment revealed global aphasia. His neurological exam showed a right 
hyperreflexia and plantar extension. Laboratorial tests were within the normal limits, except by elevated erythrocyte sedimentation rate and C-reactive protein. A cranial non-contrast computed tomography (CT) was normal.

\section{TABLE 1. Laboratory results of inflammatory markers}

\begin{tabular}{|l|c|c|}
\hline & $\begin{array}{c}\text { Erythrocyte } \\
\text { sedimenta on rate } \\
\text { (normal range less than } \\
20 \mathrm{~mm} / \mathrm{h} \text { ) }\end{array}$ & $\begin{array}{c}\text { C-reac ve protein } \\
\text { (normal range less than } \\
0.4 \mathrm{mg} / \mathrm{dl} \text { ) }\end{array}$ \\
\hline 3 & 70 & 5 \\
\hline 2.5 & 49 & 3 \\
\hline 2 & 33 & 0,7 \\
\hline 1.5 & 30 & 0,4 \\
\hline 1 & 30 & 0,4 \\
\hline 0 (stroke) & 100 & 7,2 \\
\hline
\end{tabular}

Twelve hours after admission, the patient developed a seizure, and a new non-contrast CT scan showed a hypodense area in the territory of the left middle cerebral artery (Figure 1). A computed tomography angiography revealed the hypodense area and mild left internal carotid artery stenosis without signs of narrowing, dilation, or significant dissection in other areas.



FIGURE 1. Axial view of cranial non-contrast computed tomography showing a hypodense area in the territory of the middle cerebral artery (indicated by the arrows).

Upon further questioning, the subject's family admitted that the subject reduced the dose of prednisone of $40 \mathrm{mg} /$ day to $10-20 \mathrm{mg} /$ day without any medical advice two weeks preceding the ischemic event.

\section{DISCUSSION}

GCA, also known as temporal arteritis, is a common systemic vasculitis. The clinical manifestations of GCA are the result of the inflammation from the large and medium-sized arterial walls, mainly temporal arteries. In this context, the involvement of nutrient arteries of nerves will result in the neurological symptoms as a stroke and transient ischemic attacks (TIA) (2).

A cohort study using UK Clinical Practice Research Datalink showed that subjects with GCA have more comorbidities, including stroke/TIA, before and after the diagnosis when compared with a non-vasculitis population. In the discussion, $\mathrm{Li}$ et al give some possible hypotheses for vascular diseases to be more frequently in the GCA group. The explanations included were that subclinical inflammation would occur well before the onset of GCA symptoms, some risk factors are more commonly present in the subjects diagnosed with GCA, and finally, the similarities with the pathophysiological process shared by GCA and vascular diseases (3).

The stroke is an uncommon central nervous system clinical manifestation of subjects with GCA. Most commonly occur in old males with a cardiovascular risk profile (4). The stroke territory varies between the articles, but about $80 \%$ occurs in the vertebrobasilar $(1,4)$. Other clinical stroke characteristics are the ischemic type be more common and the National Institutes of Health Stroke Scale (NIHSS) scored less than five (4).

In an Italian population-based cohort study, the risk factors associated with a higher risk of developing severe cranial ischemic events were hypertension, a history of prior ischemic heart disease (IHD), low C-reactive protein, and absence systemic manifestations. Probably atherosclerosis caused by the cardiovascular risk profile and the inflammatory process of GCA may interact increasing the risk of a severe cranial ischemic event. Already, in turn, the high inflammatory response allows an early diagnosis due to clinical manifestations and prevent severe events (5). Moreover, cerebrovascular events in individuals with GCA are probably associated with lower survival, since in a French study, GCA subjects with stroke were more likely to die than those with GCA without stroke during follow-up (6). 
The adverse outcomes of long-term use of glucocorticoids are well known. The study of Proven et al found that $86 \%$ of subjects with GCA experienced at least one adverse event during the glucocorticoid therapy. These adverse events included in descending order: posterior subcapsular cataract $(41 \%)$, fractures $(38 \%)$, infection $(31 \%)$, hypertension $(22 \%)$, diabetes mellitus $(9 \%)$, and gastrointestinal bleeding (4\%) (7). These cumulative side effects explain the importance of the glucocorticoid tapering. However, during the reducing dose of the glucocorticoid, there is $30 \%$ risk of relapse, which commonly occurs with doses of prednisone below the $20 \mathrm{mg} /$ day and is more common during the first year of treatment $(8,9)$.

The present report reinforces the importance that poor adherence to treatment and the inflammatory markers above the normal range in asymptomatic subjects possible be related to poor outcomes when the tapering dose was to fast. Since, in the

\section{REFERENCES}

1. Gonzalez-Gay MA, Vazquez-Rodriguez TR, Gomez-Acebo I, Pego-Reigosa R, Lopez-Diaz MJ, Vazquez-Triñanes MC, et al. Strokes at time of disease diagnosis in a series of 287 patients with biopsy-proven giant cell arteritis. Medicine 2009; 88:227-35.

2. Nesher G. Neurologic manifestations of giant cell arteritis. Clin Exp Rheumatol 2000;18:S-24.

3. Li L, Neogi T, Jick S. Giant cell arteritis and vascular disease-risk factors and outcomes: a cohort study using UK Clinical Practice Research Datalink. Rheumatology 2017;56:753-62.

4. Samson M, Jacquin A, Audia S, Daubail B, Devilliers H, Petrella T, et al. Stroke associated with giant cell arteritis: a population-based study. J Neurol Neurosurg Psychiatry 2015;86:216-21.

5. Salvarani C, Bella CD, Cimino L, Macchioni P, Formisano D, Bajocchi $\mathrm{G}$, et al. Risk factors for severe cranial ischaemic events in an Italian population-based cohort of patients with giant cell arteritis. Rheumatology 2008:48:250-3. present case, the subject was asymptomatic with two weeks of prednisone, however, the inflammatory markers (CRP and ESR) were above the normal range. Within 2.5 months of corticoid therapy onset, he reduced the dose of the prednisone without any medical advice and two weeks after had a stroke.

\section{CONCLUSION}

Our report suggests that stroke in GCA should be listed as a probable cause of poor therapeutic adherence. Furthermore, poor adherence to medication regimen could contribute to substantial worsening of the disease and even death. Thus, physicians should always look for poor therapeutic adherence in individuals diagnosed with GCA in order to prevent its complications.

Conflict of interest: none declared Financial support: none declared

6. Chazal T, Couture P, Rosso C, Haroche J, Léger A, Hervier B, et al. Cerebrovascular events are associated with lower survival in giant cell arteritis: a case-controlled multicenter study. Joint Bone Spine 2018;85:383-85

7. Proven A, Gabriel SE, Orces C, O'fallon WM, Hunder GG. Glucocorticoid therapy in giant cell arteritis: duration and adverse outcomes. Arthritis Rheum 2003;49:703-8.

8. Luqmani R. Discontinuation of therapies in vasculitis. Clin Exp Rheumatol 2013;31:S93-S7.

9. Visvanathan S, Rahman MU, Hoffman GS, Xu S, García-Martínez A, Segarra M, et al. Tissue and serum markers of inflammation during the follow-up of patients with giant-cell arteritis-a prospective longitudinal study. Rheumatology 2011;50:2061-70. 